NISSUNA UMANA INVESTIGAZIONE SI PUO DIMANDARE VERA SCIENZIA S'ESSA NON PASSA PER LE MATEMATICHE DIMOSTRAZIONI LEONARDO DA VINCI

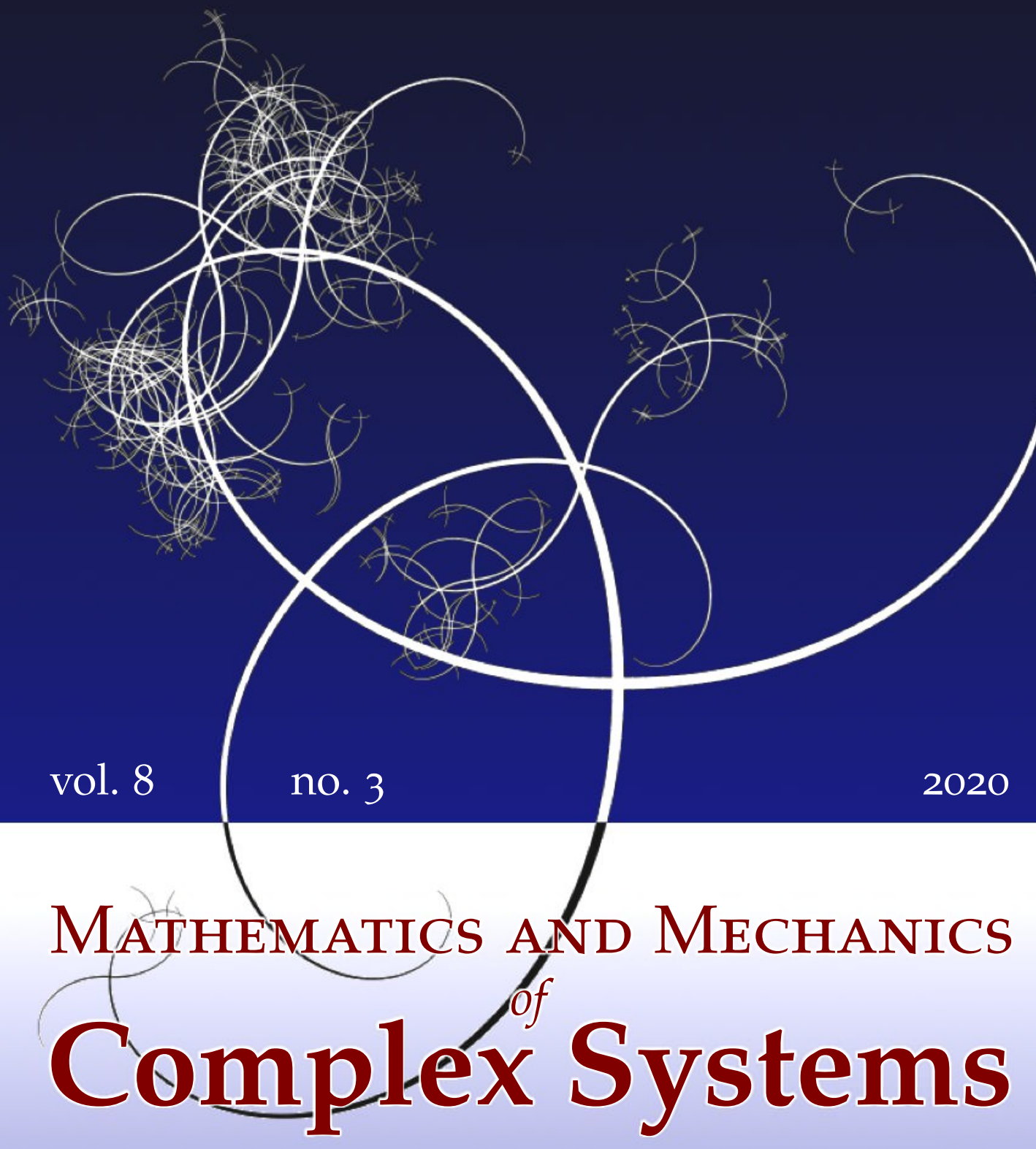

Mario Pulvirenti and Sergio Simonella

A KINETIC MODEL FOR EPIDEMIC SPREAD 


\title{
A KINETIC MODEL FOR EPIDEMIC SPREAD
}

\author{
Mario Pulvirenti And Sergio Simonella
}

We present a Boltzmann equation for mixtures of three species of particles reducing to the Kermack-McKendrick (SIR) equations for the time evolution of the density of infected agents in an isolated population. The kinetic model is potentially more detailed and might provide information on space mixing of the agents.

\section{Boltzmann-SIR equations}

Consider a population of identical individuals (particles) moving in physical space and interacting upon contact. One (or several) of the individuals, say particle 1, has an infected status at time zero. As the dynamics runs, the infection can be transmitted, at the interaction times, to the individuals entering in contact with 1 or with the newly infected individuals. A cluster $\left\{i_{1}, i_{2}, \ldots\right\}$ of infection grows in time, determined by the particle evolution: an individual is potentially infected at time $t>0$ if it is involved, directly or indirectly, in the forward-in-time dynamics of 1. The "forward cluster of particle 1" (in the terminology of [Aoki et al. 2015; Pulvirenti and Simonella 2020b]) is represented symbolically in the picture below:

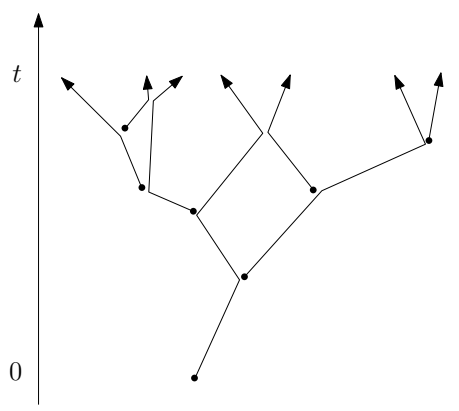

For concreteness, we may want to fix an idealized mechanical setting. Let us then proceed, as is customary in kinetic theory, by looking at $N$ hard spheres of unit mass and diameter $\varepsilon>0$. The balls move in $\Lambda \subset \mathbb{R}^{d}, d=2,3$, and interact

\section{Communicated by Raffaele Esposito.}

MSC2020: 00A71.

Keywords: Boltzmann equation, SIR model, low-density limit, stochastic particle system, forward cluster. 
through elastic collisions. Each particle flies freely with constant velocity, and when two hard spheres collide with positions $x, x_{*}$ at distance $\varepsilon$ and incoming velocities $v, v_{*}$, the latter are instantaneously transformed to outgoing velocities $v^{\prime}, v_{*}^{\prime}$ by the relations

$$
\left\{\begin{array}{c}
v^{\prime}=v-\omega\left[\omega \cdot\left(v-v_{*}\right)\right] \\
v_{*}^{\prime}=v_{*}+\omega\left[\omega \cdot\left(v-v_{*}\right)\right]
\end{array}\right.
$$

where $\omega$ is the normalized relative distance $\omega=\left(x-x_{*}\right) /\left|x-x_{*}\right|=\left(x-x_{*}\right) / \varepsilon \in \mathbb{S}^{d-1}$.

We shall mimic the basic model in the mathematical theory of epidemics [Kermack and McKendrick 1927], by means of several assumptions. There are three different species of particles, $S, I$, and $R$, which stand for susceptible, infected, and recovered, respectively. Upon collision between a particle of type $S$ and a particle of type $I$, the reaction

$$
S+I \rightarrow I+I
$$

occurs instantaneously with rate $\beta \in[0,1]$. All the other collisions do not change the particle type, but in addition, a decay

$$
I \rightarrow R
$$

occurs with rate $\gamma \in[0,1]$. Note that the population size is fixed (no deaths) and that the infection implies complete immunity. Finally for simplicity, we shall assume that $\beta$ and $\gamma$ are constants (they do not depend on time).

We are relying on the idea that the details of the interactions should not be of crucial importance (see [Stevens 2020] for a recent popular article simulating a similar system of particles). The main features are instead the following:

- The interactions are binary and localized.

- The number of interactions per unit time is expected to be finite.

- The qualitative behavior is independent of the number of particles $N$, provided that this is large in a suitable scaling limit.

- A statistical description is appropriate.

Under these assumptions, the Boltzmann equation for rarefied gases provides a tool of investigation.

Let us perform the so-called Boltzmann-Grad limit [Grad 1949] on the hardsphere system under consideration. Denoting the one-particle distribution functions by

$$
\begin{aligned}
f_{S} & =f_{S}(t, x, v), \\
f_{I} & =f_{I}(t, x, v), \\
f_{R} & =f_{R}(t, x, v)
\end{aligned}
$$


for the three species of particles, we obtain the set of equations

$$
\left\{\begin{array}{l}
\left(\partial_{t}+v \cdot \nabla_{x}\right) f_{S}=Q\left(f_{S}, f_{S}\right)+Q\left(f_{S}, f_{R}\right)+(1-\beta) Q\left(f_{S}, f_{I}\right)-\beta Q_{-}\left(f_{S}, f_{I}\right) \\
\left(\partial_{t}+v \cdot \nabla_{x}\right) f_{I}=Q\left(f_{I}, f\right)+\beta Q_{+}\left(f_{S}, f_{I}\right)-\gamma f_{I} \\
\left(\partial_{t}+v \cdot \nabla_{x}\right) f_{R}=Q\left(f_{R}, f\right)+\gamma f_{I}
\end{array}\right.
$$

where

$$
f=f_{S}+f_{I}+f_{R}
$$

and $Q$ is Boltzmann's operator (expressed in asymmetric form)

$$
\begin{aligned}
Q & =Q_{+}-Q_{-}, \\
Q_{+}(f, g)(v) & :=\int_{\mathbb{R}^{d}} \int_{\mathbb{S}^{d-1}} B\left(\omega ; v-v_{*}\right) f\left(v^{\prime}\right) g\left(v_{*}^{\prime}\right) d \omega d v_{*}, \\
Q_{-}(f, g)(v) & :=f(v) \int_{\mathbb{R}^{d}} \int_{\mathbb{S}^{d-1}} B\left(\omega ; v-v_{*}\right) g\left(v_{*}\right) d \omega d v_{*} .
\end{aligned}
$$

Note that the sum $f=f_{S}+f_{I}+f_{R}$ satisfies the classical Boltzmann equation

$$
\left(\partial_{t}+v \cdot \nabla_{x}\right) f=Q(f, f) .
$$

Here we chose $B\left(\omega ; v-v_{*}\right)=\left(\omega \cdot\left(v-v_{*}\right)\right) \mathbb{1}\left(\omega \cdot\left(v-v_{*}\right) \geq 0\right)$, corresponding to the hard-sphere cross section. However, as said above, conclusions drawn from the kinetic model should not be very sensitive to the interaction rule; e.g., we shall consider as well different kernels $B\left(\omega ; v-v_{*}\right) \geq 0$ such that

$$
\int_{\mathbb{S}^{d-1}} B\left(\omega ; v-v_{*}\right) d \omega=\left|v-v_{*}\right|^{b}
$$

for some $b \geq 0$.

In the second part of this exposition we will give more details on the passage from the particle dynamics to (1-2). Before that, we make a few elementary remarks on the equations themselves.

1.1. Maxwell collisions: Kermack-McKendrick equations. Averaging (1-2) over velocities, the $Q$ operators vanish (because $\int Q_{+}=\int Q_{-}$), and in the spatially homogeneous case (no dependence on $x$ ), the expected fractions of individuals of the species $A \in\{S, I, R\}, A(t)=\int f_{A}(t, v) d v$, satisfy the equations

$$
\left\{\begin{array}{l}
\dot{S}=-\beta \int\left|v-v_{*}\right|^{b} f_{S}(v) f_{I}\left(v_{*}\right) d v d v_{*} \\
\dot{I}=\beta \int\left|v-v_{*}\right|^{b} f_{S}(v) f_{I}\left(v_{*}\right) d v d v_{*}-\gamma I \\
\dot{R}=\gamma I
\end{array}\right.
$$


These equations are not closed, except when dealing with "Maxwellian molecules" (case $b=0$ [Bobylev 1988]), for which we get

$$
\left\{\begin{array}{l}
\dot{S}=-\beta I S, \\
\dot{I}=\beta S I-\gamma I, \\
\dot{R}=\gamma I,
\end{array}\right.
$$

namely the epidemiology model of [Kermack and McKendrick 1927] in the case of time-independent rates. This model has been analyzed and used extensively, and several generalizations have been conceived; see, e.g., [Anderson and May 1979; Brauer and Castillo-Chávez 2001; Murray 2002; Harko et al. 2014]. The kinetic equation (1-2) stands as an extension accounting for dependence on space and velocity of the individuals.

To remind the reader of the original motivations for such SIR models [Ross 1916; Kermack and McKendrick 1927], we recall that an epidemic is not necessarily terminated by the exhaustion of the susceptible individuals, nor by the extinction of the virulence. This is apparent from (1-3), over a threshold value of the density. Setting indeed $A_{\infty}:=\lim _{t \rightarrow \infty} A(t), A_{0}=A(0)$, and $R(t)=R_{0}+\gamma \int_{0}^{t} I(\tau) d \tau$ (showing that $I(t) \rightarrow 0$ as $t \rightarrow \infty)$, one has that $\frac{d S}{d R}=-(\beta / \gamma) S$ and hence (by $R_{\infty}+S_{\infty}=1$ and the assumption $\left.R_{0}=0\right) S_{\infty}=S_{0} e^{-(\beta / \gamma)\left(1-S_{\infty}\right)}$, or

$$
e^{-(\beta / \gamma) S_{\infty}} \frac{\beta}{\gamma} S_{\infty}=S_{0} \frac{\beta}{\gamma} e^{-\beta / \gamma}
$$

Since max $y e^{-y}=1 / e$, given a value of $\beta / \gamma$ one can find nonvanishing solutions for $S_{\infty}$.

1.2. Confinement. The model can be easily adapted to investigate several different situations. Examples might be boundary conditions or external potentials, imposing internal spatial constraints or local enhancing of density. There has been recent intense interest in the effects of isolation of individuals, and of the reduction of social mixing, by means of physical distancing measures [Li et al. 2020; Prem et al. 2020]. At the level of (1-2), the energy can be used as a simple parameter regulating the interaction rate.

Here we give an example of one adaptation of (1-2), intended to model a confinement effect. Following [Stevens 2020] we assume that, for each species, there are two types of particles: wandering and confined. We denote by $g_{A}, A \in\{S, I, R\}$, the distribution of confined particles, while we maintain the notation $f_{A}$ for the wandering particles. The distribution of the species $A$ is $h_{A}:=f_{A}+g_{A}$ and $f=\sum_{A} h_{A}$. Wandering particles have mass $m_{w}=1$, while confined particles have mass $m_{c}=+\infty$ and zero velocity. The distribution $g_{A}$ is proportional to a Dirac delta in velocity. Confined particles are frozen, and their total distribution is 
stationary:

$$
g_{S}(t, x)+g_{I}(t, x)+g_{R}(t, x)=\text { const. for all } t .
$$

The collision law becomes

$$
\left\{\begin{array}{l}
v^{\prime}=v-\left(2 m_{*} /\left(m+m_{*}\right)\right) \omega\left[\omega \cdot\left(v-v_{*}\right)\right], \\
v_{*}^{\prime}=v_{*}+\left(2 m /\left(m+m_{*}\right)\right) \omega\left[\omega \cdot\left(v-v_{*}\right)\right],
\end{array}\right.
$$

where $m, m_{*}$ are the masses of the incoming particles, and (1-2) is replaced by

$$
\left\{\begin{aligned}
\left(\partial_{t}+v \cdot \nabla_{x}\right) f_{S} & =Q\left(f_{S}, h_{S}\right)+Q\left(f_{S}, h_{R}\right)+(1-\beta) Q\left(f_{S}, h_{I}\right)-\beta Q_{-}\left(f_{S}, h_{I}\right), \\
\left(\partial_{t}+v \cdot \nabla_{x}\right) f_{I} & =Q\left(f_{I}, f\right)+\beta Q_{+}\left(f_{S}, h_{I}\right)-\gamma f_{I}, \\
\left(\partial_{t}+v \cdot \nabla_{x}\right) f_{R} & =Q\left(f_{R}, f\right)+\gamma f_{I}, \\
\dot{g_{S}} & =-\beta Q_{-}\left(g_{S}, f_{I}\right), \\
\dot{g_{I}} & =\beta Q_{+}\left(g_{S}, f_{I}\right)-\gamma g_{I}, \\
\dot{g_{R}} & =\gamma g_{I} .
\end{aligned}\right.
$$

In the spatially homogeneous case, integrating (1-5) in $v$, calling $A_{w}=\int f_{A} d v$ and $A_{c}=\int g_{A} d v, A=S, I, R$, we obtain

$$
\left\{\begin{array}{l}
\dot{S}_{w}=-\beta \int\left|v-v_{*}\right|^{b} f_{S}(v) h_{I}\left(v_{*}\right) d v d v_{*} \\
\dot{I}_{w}=\beta \int\left|v-v_{*}\right|^{b} f_{S}(v) h_{I}\left(v_{*}\right) d v d v_{*}-\gamma I_{w} \\
\dot{R}_{w}=\gamma I_{w} \\
\dot{S}_{c}=-S_{c} \int\left|v_{*}\right|^{b} f_{I}\left(v_{*}\right) d v_{*} \\
\dot{I}_{c}=S_{c} \int\left|v_{*}\right|^{b} f_{I}\left(v_{*}\right) d v_{*}-\gamma I_{c} \\
\dot{R}_{c}=\gamma I_{c} .
\end{array}\right.
$$

Again, the above equations reduce to a standard SIR model in the case of Maxwellian molecules:

$$
\left\{\begin{aligned}
\dot{S}_{w} & =-\beta S_{w}\left(I_{w}+I_{c}\right) \\
\dot{I}_{w} & =\beta S_{w}\left(I_{w}+I_{c}\right)-\gamma I_{w} \\
\dot{R}_{w} & =\gamma I_{w} \\
\dot{S}_{c} & =-S_{c} I_{w} \\
\dot{I}_{c} & =S_{c} I_{w}-\gamma I_{c} \\
\dot{R}_{c} & =\gamma I_{c}
\end{aligned}\right.
$$

1.3. Related problems. The kinetic model presented above should be interpreted as a remark in the vein of mathematical physics: we do not pretend that it can be of use in epidemiology. It is more detailed than the classical SIR, insofar as it includes space and velocities of the agents. Presumably, its main potential interest in applications is the identification of spatial patterns having an impact on the history of epidemics. Moreover, a dynamical representation in terms of forward (or backward) clusters would provide information on the tracing of the infection. We comment next on a few other problems arising naturally. 
The typical question concerning SIR equations is determining the long-time behavior in relation with the parameters $\beta, \gamma$ and its dependence on local characteristics of the initial data. We are interested in masses but also in local densities in the presence of spatial inhomogeneities. From the mathematical side, little can be done, but the problem is suited to numerical investigation. In analogy to gas dynamics, it is natural to use stochastic methods, as we will discuss in the next section.

At the theoretical level, it would be interesting to detect large-scale limits and derive, starting from (1-2), equations for locally conserved quantities. Equation (1-2) can be useful in fact for limited amounts of time. Preliminarily, one should characterize the equilibria. Let $F_{A}=\lim _{t \rightarrow 0} f_{A}$ be the asymptotic distributions. Then we expect $F_{I}=0$, and the other two distributions should satisfy

$$
\left\{\begin{array}{l}
Q\left(F_{S}, F_{S}\right)+Q\left(F_{S}, F_{R}\right)=0 \\
Q\left(F_{R}, F_{R}\right)+Q\left(F_{R}, F_{S}\right)=0 .
\end{array}\right.
$$

The latter equation is satisfied if both $F_{S}$ and $F_{R}$ are Maxwellians

$$
F_{A}=A_{\infty} \frac{e^{-(v-u)^{2} /\left(2 \sigma^{2}\right)}}{\left(2 \pi \sigma^{2}\right)^{d / 2}}
$$

for some constants $S_{\infty}$ and $R_{\infty}$, with $\sigma$ and $u$ determined by the initial conditions. $A_{\infty}$ would be obtained as in (1-4). Notice that, when $f=f_{S}+f_{I}+f_{R}$ is a global equilibrium, a solution $\left(f_{S}, f_{I}, f_{R}\right)$ of (1-2) for $b=0$ is given by the same global equilibrium with densities $S(t), I(t), R(t)$ driven by (1-3).

\section{Particle systems}

2.1. Stochastic particle system. In this section we introduce a particle system yielding, in a suitable scaling limit, kinetic equations of type (1-2). The interest of this dynamics is twofold. First, it can be considered a microscopic model to be accepted through phenomenology, covering a large variety of kernels $B$. It would be somewhat funny to believe that the laws of Newton can be used to efficiently describe the interaction among individuals. On the other hand, we do not know so much concerning the details of such interactions; thus, a stochastic collision appears to be more robust than a deterministic one. Secondly, the particle scheme corresponds numerically to the direct simulation Monte Carlo method, widely used to approximate rarefied gas dynamics. There are several variants of such methods [Bird 1994; Rjasanow and Wagner 2005]. Below, we will deal with an inhomogeneous Kac model [1956] for three species with reactions. 
We start by regularizing the collision operator (1-2). The strictly local interaction is smeared as

$$
\begin{aligned}
Q^{h} & =Q_{+}^{h}-Q_{-}^{h}, \\
Q_{+}^{h}(f, g)(x, v) & :=\int_{\mathbb{R}^{d}} \int_{\mathbb{R}^{d}} \int_{\mathbb{S}^{d-1}} B\left(\omega ; v-v_{*}\right) h(|x-y|) f\left(x, v^{\prime}\right) g\left(y, v_{*}^{\prime}\right) d \omega d v_{*} d y, \\
Q_{-}^{h}(f, g)(x, v) & :=\int_{\mathbb{R}^{d}} \int_{\mathbb{R}^{d}} \int_{\mathbb{S}^{d-1}} B\left(\omega ; v-v_{*}\right) h(|x-y|) f(x, v) g\left(y, v_{*}\right) d \omega d v_{*} d y,
\end{aligned}
$$

where $h: \mathbb{R}^{+} \rightarrow \mathbb{R}^{+}$is a smooth approximation of the delta function.

To simplify the notation, we limit ourselves to the case of (1-2) with $\beta=1$, with the more general cases being a trivial extension. We therefore consider

$$
\left\{\begin{array}{l}
\left(\partial_{t}+v \cdot \nabla_{x}\right) f_{S}=Q^{h}\left(f_{S}, f_{S}\right)+Q^{h}\left(f_{S}, f_{R}\right)-Q_{-}^{h}\left(f_{S}, f_{I}\right), \\
\left(\partial_{t}+v \cdot \nabla_{x}\right) f_{I}=Q^{h}\left(f_{I}, f\right)+Q_{+}^{h}\left(f_{S}, f_{I}\right)-\gamma f_{I}, \\
\left(\partial_{t}+v \cdot \nabla_{x}\right) f_{R}=Q^{h}\left(f_{R}, f\right)+\gamma f_{I} .
\end{array}\right.
$$

We can pass to the limit $Q^{h} \rightarrow Q$ inside (2-1), whenever we have a smooth solution of the initial value problem.

We shall indicate by $\mathscr{A}=\mathscr{Y}, \mathscr{I}, \mathscr{R} \subset\{1,2, \ldots, N\}$ the (random) disjoint sets of particles of types $A=S, I, R$, respectively. They form a partition of $\{1,2, \ldots, N\}$, so that the process $Z_{N}: \mathbb{R}^{+} \rightarrow \mathscr{X}, Z_{N}=Z_{N}(t)=\left(z_{1}(t), \ldots, z_{N}(t)\right), z_{i}=\left(x_{i}, v_{i}\right)$, takes values in

$$
\mathscr{X}=\bigcup_{\mathscr{S}, \mathscr{I}, \mathscr{R}} \mathscr{X}(\mathscr{Y}, \mathscr{I}, \mathscr{R}), \quad \mathscr{X}(\mathscr{Y}, \mathscr{I}, \mathscr{R})=\left\{\left(Z_{\mathscr{Y}}, Z_{\mathscr{I}}, Z_{\mathscr{R}}\right)\right\},
$$

with

$$
|\mathscr{Y}|+|\mathscr{I}|+|\mathscr{R}|=N
$$

and $z_{i} \in \Lambda \times \mathbb{R}^{d}$. Here $|\mathscr{A}|$ denotes the cardinality of the set $\mathscr{A}$. The configurations of particles in the three species are $Z_{\mathscr{S}}=\left(z_{s_{1}}, z_{s_{2}}, \ldots\right), Z_{\mathscr{I}}=\left(z_{i_{1}}, z_{i_{2}}, \ldots\right)$, and $Z_{\overparen{R}}=\left(z_{r_{1}}, z_{r_{2}}, \ldots\right)$, respectively.

Le us define the time evolution. Particles move freely for a random time, exponentially distributed with intensity scaling like $N$. Then two particles are randomly chosen, say particles $j$ and $k$, according to $\int B\left(\omega ; v_{j}-v_{k}\right) h\left(\left|x_{j}-x_{k}\right|\right) d \omega$, and their velocities are updated as in (1-1) with $\omega \sim B\left(\cdot ; v_{j}-v_{k}\right)$. If the pair of colliding particles is of type $(A, A)$ or $(S, R)$ or $(I, R)$, the particles do not change their species. If the pair is of type $(S, I)$, then the outgoing pair is of type $(I, I)$. We abbreviate from now on $h_{j, k}=h\left(\left|x_{j}-x_{k}\right|\right)$, and we denote by $J_{j k}$ the linear operator transforming the velocities $j$ and $k$ to a postcollisional pair with scattering vector $\omega$. The generator of the process reads

$$
\mathscr{L}=\mathscr{L}_{0}+\mathscr{L}_{i}+\mathscr{L}_{d}
$$


where $\mathscr{L}_{0}=\sum v_{i} \cdot \nabla_{x_{i}}$ is the generator of the free motion,

$$
\begin{aligned}
& \mathscr{L}_{i} \phi\left(Z_{N}\right)=\frac{1}{N} \sum_{j \in \mathscr{S}} \sum_{k \in \mathscr{I}} \int B\left(\omega ; v_{j}-v_{k}\right) h_{j, k} \\
& \times\left(J_{j k} \phi\left(Z_{\mathscr{S} \backslash j\}}, Z_{\mathscr{g} \cup\{j\}}, Z_{\mathscr{R}}\right)-\phi\left(Z_{N}\right)\right) d \omega \\
& +\frac{1}{N}\left(\sum_{j \in \mathscr{S}} \sum_{k \in \mathscr{R}}+\sum_{j \in \mathscr{I}} \sum_{k \in \mathscr{R}}\right) \int B\left(\omega ; v_{j}-v_{k}\right) h_{j, k}\left(J_{j k} \phi\left(Z_{N}\right)-\phi\left(Z_{N}\right)\right) d \omega \\
& +\frac{1}{2 N} \sum_{\mathscr{A}=\mathscr{G}, \mathscr{I}, \mathscr{R}} \sum_{\substack{j, k \in \mathcal{A} \\
j \neq k}} \int B\left(\omega ; v_{j}-v_{k}\right) h_{j, k}\left(J_{j k} \phi\left(Z_{N}\right)-\phi\left(Z_{N}\right)\right) d \omega,
\end{aligned}
$$

and

$$
\mathscr{L}_{d} \phi\left(Z_{N}\right)=\gamma \sum_{i \in \mathscr{I}}\left(\phi\left(Z_{\mathscr{S}}, Z_{\mathscr{I} \backslash\{i\}}, Z_{\mathscr{R} \cup\{i\}}\right)-\phi\left(Z_{N}\right)\right) .
$$

We choose now test functions of the form

$$
\phi_{A}\left(Z_{N}\right)=\frac{1}{N} \sum_{\ell \in \mathscr{A}} \varphi\left(z_{\ell}\right)
$$

and focus, for instance, on the case $\mathscr{A}=\mathscr{Y}$. We have that $\mathscr{L}_{d} \phi_{S}=0$. Evaluating (2-2) in $\phi_{S}$ we notice that, given $j$ and $k$, all the terms with $\ell \neq j, k$ cancel out. In the second line of (2-2) we find

$$
\sum_{\substack{\ell \in \mathcal{S} \\ \ell \neq j}} J_{j k} \varphi\left(z_{\ell}\right)-\sum_{\ell \in \mathscr{S}} \varphi\left(z_{\ell}\right)=-\varphi\left(z_{j}\right) .
$$

Therefore,

$$
\begin{aligned}
& \mathscr{L}_{i} \phi_{S}\left(Z_{N}\right)=-\frac{1}{N^{2}} \sum_{j \in \mathscr{S}} \sum_{k \in \mathscr{I}} \int B\left(\omega ; v_{j}-v_{k}\right) h_{j, k} \varphi\left(z_{j}\right) d \omega \\
& +\frac{1}{N^{2}} \sum_{j \in \mathscr{T}} \sum_{k \in \mathscr{R}} \int B\left(\omega ; v_{j}-v_{k}\right) h_{j, k}\left(\varphi\left(x_{j}, v_{j}^{\prime}\right)-\varphi\left(z_{j}\right)\right) d \omega \\
& +\frac{1}{2 N^{2}} \sum_{\substack{j, k \in \mathscr{S} \\
j \neq k}} \int B\left(\omega ; v_{j}-v_{k}\right) h_{j, k}\left(\varphi\left(x_{j}, v_{j}^{\prime}\right)+\varphi\left(x_{k}, v_{k}^{\prime}\right)-\varphi\left(z_{j}\right)-\varphi\left(z_{k}\right)\right) d \omega .
\end{aligned}
$$

Next, we introduce a probability measure with density $W^{N}: \mathscr{L} \rightarrow \mathbb{R}^{+}$, assumed to be symmetric in the exchange of the particle labels within each one of the species. An example is provided by the fully factorized (chaotic) state, which we shall assume, to fix ideas, as the initial distribution of the particle process: $W^{N}(0)=f_{0}^{\otimes N}$ with $f^{0}=\sum_{A} f_{A}^{0}, A=(S, I, R)$, where $f_{A}^{0}$ are the initial data for (2-1). We further 
denote by $f_{A}^{N}=f_{A}^{N}(z)$ the one-particle marginals of $W^{N}$, defined as

$$
\int f_{A}^{N}(z) \varphi(z) d z=\int W^{N}\left(Z_{N}\right) \phi_{A}\left(Z_{N}\right) d Z_{N}
$$

It is the probability density of finding a particle of type $A$ in $z$. Similarly, $f_{A_{1}, A_{2}}^{N}=$ $f_{A_{1}, A_{2}}^{N}\left(z_{1}, z_{2}\right)$ denotes the two-particle marginal, namely the probability density of finding two particles of type $A_{1}$ and $A_{2}$ in $z_{1}$ and $z_{2}$ :

$$
\int f_{A_{1}, A_{2}}^{N}\left(z_{1}, z_{2}\right) \varphi\left(z_{1}, z_{2}\right) d z_{1} d z_{2}=\int W^{N}\left(Z_{N}\right) \phi_{A_{1}, A_{2}}\left(Z_{N}\right) d Z_{N}
$$

for

$$
\phi_{A_{1}, A_{2}}\left(Z_{N}\right)=\frac{1}{N(N-1)} \sum_{j \in \mathscr{A}_{1}} \sum_{\substack{k \in \mathscr{A}_{2} \\ k \neq j}} \varphi\left(z_{j}, z_{k}\right)
$$

Even though the initial measure is factorized, the time-evolved density $W^{N}(t)$ is not, due to correlations generated by the dynamics. The factorization is however recovered in the limit $N \rightarrow \infty$ and

$$
f_{A_{1}, A_{2}}^{N}\left(z_{1}, z_{2}\right) \approx f_{A_{1}}^{N}\left(z_{1}\right) f_{A_{2}}^{N}\left(z_{2}\right)
$$

We are ready to compute

$$
\frac{d}{d t} \int W^{N}(t) \phi_{S}=\int W^{N}(t) \mathscr{L} \phi_{S} .
$$

Using (2-4), the definition of marginal, and (2-5), we deduce that, as $N \rightarrow \infty$,

$$
\begin{aligned}
\frac{d}{d t} \int f_{S}^{N}(t) \varphi \approx \int f_{S}^{N}\left(v \cdot \nabla_{x} \varphi\right)+\int Q^{h}\left(f_{S}^{N}, f_{S}^{N}\right) \varphi & \\
& +\int Q^{h}\left(f_{S}^{N}, f_{R}^{N}\right) \varphi-\int Q_{-}^{h}\left(f_{S}^{N}, f_{I}^{N}\right) \varphi,
\end{aligned}
$$

that is, the first equation of (2-1) in weak formulation.

The other two equations can be recovered similarly. For $A=I,(2-3)$ yields

$$
\mathscr{L}_{d} \phi_{I}\left(Z_{N}\right)=\frac{\gamma}{N} \sum_{i \in \mathscr{I}}\left(\sum_{\ell \in \mathscr{I} \backslash\{i\}} \varphi\left(z_{\ell}\right)-\sum_{\ell \in \mathscr{I}} \varphi\left(z_{\ell}\right)\right)=-\frac{\gamma}{N} \sum_{i \in \mathscr{I}} \varphi\left(z_{i}\right),
$$

while in the second line of $(2-2)$ we find

$$
\sum_{\ell \in \mathscr{I} \cup\{j\}} J_{j k} \varphi\left(z_{\ell}\right)-\sum_{\ell \in \mathscr{I}} \varphi\left(z_{\ell}\right)=J_{j, k} \varphi\left(z_{j}\right)+\left(J_{j, k} \varphi\left(z_{k}\right)-\varphi\left(z_{k}\right)\right)
$$


so that

$$
\begin{aligned}
\frac{d}{d t} \int f_{I}^{N}(t) \approx \int f_{I}^{N}\left(v \cdot \nabla_{x} \varphi\right)+\int Q^{h}\left(f_{I}^{N}, \sum_{A} f_{A}^{N}\right) \varphi & \\
& +\int Q_{+}^{h}\left(f_{S}^{N}, f_{I}^{N}\right) \varphi-\gamma \int f_{I}^{N} \varphi,
\end{aligned}
$$

which is the second equation of (2-1).

2.2. Mechanical system. We briefly come back to the deterministic particle model, which was our starting point, that is, $N$ hard spheres of diameter $\varepsilon$ moving in physical space and colliding elastically, with reactions simulating infection and recovery. We call this system "mechanical" as the interaction is deterministic. Clearly there is still stochasticity in the reactions and, strictly speaking, we are dealing again with a stochastic process.

We can easily adapt to this case the formal arguments of the previous section. The process $Z_{N}$ still takes values in $\mathscr{X}$, but in addition the strict exclusion $\min _{i \neq j}\left|x_{i}-x_{j}\right|>\varepsilon$ is imposed. In the generator (2-2), $1 / N$ is replaced by $\varepsilon^{d-1}$, $B$ is the hard-sphere kernel $\left(\omega \cdot\left(v_{j}-v_{k}\right)\right) \mathbb{1}\left(\omega \cdot\left(v_{j}-v_{k}\right) \geq 0\right), h_{j, k}$ is absent, and the operator $\left(J_{j, k}-1\right)$ is replaced by $\left(\delta\left(x_{k}-x_{j}-\omega \varepsilon\right) J_{j, k}-\delta\left(x_{k}-x_{j}+\omega \varepsilon\right)\right)$. Following [Pulvirenti and Simonella 2020a, §2.1] and assuming the chaos property (2-5), (1-2) is obtained in the limit $N \rightarrow \infty, \varepsilon \rightarrow 0$ with $\varepsilon^{d-1} N=1$.

2.3. Rigorous results. We have formally derived the kinetic equations under proper scaling limits, presenting only the basic ideas. A rigorous approach is possible, based on existing literature. In the case of the stochastic system, one can apply martingale techniques as in [Wagner 1992], or the hierarchy of equations for the family of the marginals [Pulvirenti et al. 1994], or coupling techniques [Graham and Méléard 1997]. In the case of the mechanical model, one can resort to the validity techniques for the Boltzmann equation, leading to a short-time result; see [Lanford 1975] and subsequent works [Illner and Pulvirenti 1989; Spohn 1991; Cercignani et al. 1994; Gallagher et al. 2013; Pulvirenti et al. 2014; Pulvirenti and Simonella 2017; Denlinger 2018].

\section{Acknowledgments}

We are indebted to Grégory Miermont for enlightening comments inspiring this work. We thank Nicola Cotugno for advice on the bibliography and Robert Patterson for sharing numerical simulations and for useful comments on the manuscript.

\section{References}

[Anderson and May 1979] R. M. Anderson and R. M. May, "Population biology of infectious diseases, I", Nature 280 (1979), 361-367. 
[Aoki et al. 2015] K. Aoki, M. Pulvirenti, S. Simonella, and T. Tsuji, "Backward clusters, hierarchy and wild sums for a hard sphere system in a low-density regime", Math. Models Methods Appl. Sci. 25:5 (2015), 995-1010.

[Bird 1994] G. A. Bird, Molecular gas dynamics and the direct simulation of gas flows, Oxford Engineering Science Series 42, Clarendon, Oxford, 1994.

[Bobylev 1988] A. V. Bobylev, "The theory of the nonlinear spatially uniform Boltzmann equation for Maxwell molecules", Soviet Sci. Rev. C Math. Phys. Rev. 7 (1988), 111-233.

[Brauer and Castillo-Chávez 2001] F. Brauer and C. Castillo-Chávez, Mathematical models in population biology and epidemiology, Texts in Applied Mathematics 40, Springer, 2001.

[Cercignani et al. 1994] C. Cercignani, R. Illner, and M. Pulvirenti, The mathematical theory of dilute gases, Applied Mathematical Sciences 106, Springer, 1994.

[Denlinger 2018] R. Denlinger, "The propagation of chaos for a rarefied gas of hard spheres in the whole space", Arch. Ration. Mech. Anal. 229:2 (2018), 885-952.

[Gallagher et al. 2013] I. Gallagher, L. Saint-Raymond, and B. Texier, From Newton to Boltzmann: hard spheres and short-range potentials, Zurich Lectures in Advanced Mathematics 18, European Mathematical Society, Zürich, 2013.

[Grad 1949] H. Grad, "On the kinetic theory of rarefied gases", Comm. Pure Appl. Math. 2 (1949), 331-407.

[Graham and Méléard 1997] C. Graham and S. Méléard, "Stochastic particle approximations for generalized Boltzmann models and convergence estimates", Ann. Probab. 25:1 (1997), 115-132.

[Harko et al. 2014] T. Harko, F. S. N. Lobo, and M. K. Mak, "Exact analytical solutions of the susceptible-infected-recovered (SIR) epidemic model and of the SIR model with equal death and birth rates", Appl. Math. Comput. 236 (2014), 184-194.

[Illner and Pulvirenti 1989] R. Illner and M. Pulvirenti, "Global validity of the Boltzmann equation for two- and three-dimensional rare gas in vacuum: erratum and improved result", Comm. Math. Phys. 121:1 (1989), 143-146.

[Kac 1956] M. Kac, "Foundations of kinetic theory", pp. 171-197 in Proceedings of the Third Berkeley Symposium on Mathematical Statistics and Probability (Berkeley, CA, 1954-1955), vol. III: Contributions to astronomy and physics, edited by J. Neyman, University of California, Berkeley, CA, 1956.

[Kermack and McKendrick 1927] W. O. Kermack and A. G. McKendrick, "A contribution to the mathematical theory of epidemics", Proc. Roy. Soc. Lond. A 115:772 (1927), 700-721.

[Lanford 1975] O. E. Lanford, III, "Time evolution of large classical systems", pp. 1-111 in Dynamical systems, theory and applications (Seattle, 1974), edited by J. Moser, Lecture Notes in Physcs 38, Springer, 1975.

[Li et al. 2020] Q. Li, X. Guan, P. Wu, X. Wang, L. Zhou, Y. Tong, R. Ren, K. S. M. Leung, E. H. Y. Lau, J. Y. Wong, X. Xing, N. Xiang, Y. Wu, C. Li, Q. Chen, D. Li, T. Liu, J. Zhao, M. Liu, W. Tu, C. Chen, L. Jin, R. Yang, Q. Wang, S. Zhou, R. Wang, H. Liu, Y. Luo, Y. Liu, G. Shao, H. Li, Z. Tao, Y. Yang, Z. Deng, B. Liu, Z. Ma, Y. Zhang, G. Shi, T. T. Y. Lam, J. T. Wu, G. F. Gao, B. J. Cowling, B. Yang, G. M. Leung, and Z. Feng, "Early transmission dynamics in Wuhan, China, of novel coronavirus-infected pneumonia", New Engl. J. Med. 382:13 (2020), 1199-1207.

[Murray 2002] J. D. Murray, Mathematical biology, vol. I: An introduction, 3rd ed., Interdisciplinary Applied Mathematics 17, Springer, 2002.

[Prem et al. 2020] K. Prem, Y. Liu, T. W. Russell, A. J. Kucharski, R. M. Eggo, N. Davies, Centre for the Mathematical Modelling of Infectious Diseases COVID-19 Working Group, M. Jit, and P. 
Klepac, "The effect of control strategies to reduce social mixing on outcomes of the COVID-19 epidemic in Wuhan, China: a modelling study", Lancet Publ. Health 5:5 (2020), E261-E270.

[Pulvirenti and Simonella 2017] M. Pulvirenti and S. Simonella, "The Boltzmann-Grad limit of a hard sphere system: analysis of the correlation error", Invent. Math. 207:3 (2017), 1135-1237.

[Pulvirenti and Simonella 2020a] M. Pulvirenti and S. Simonella, "A brief introduction to the scaling limits and effective equations in kinetic theory", preprint, 2020. arXiv

[Pulvirenti and Simonella 2020b] M. Pulvirenti and S. Simonella, "On the cardinality of collisional clusters for hard spheres at low density", preprint, 2020. arXiv

[Pulvirenti et al. 1994] M. Pulvirenti, W. Wagner, and M. B. Zavelani Rossi, "Convergence of particle schemes for the Boltzmann equation”, European J. Mech. B Fluids 13:3 (1994), 339-351.

[Pulvirenti et al. 2014] M. Pulvirenti, C. Saffirio, and S. Simonella, "On the validity of the Boltzmann equation for short range potentials", Rev. Math. Phys. 26:2 (2014), art. id. 1450001.

[Rjasanow and Wagner 2005] S. Rjasanow and W. Wagner, Stochastic numerics for the Boltzmann equation, Springer Series in Computational Mathematics 37, Springer, 2005.

[Ross 1916] R. Ross, "An application of the theory of probabilities to the study of a priori pathometry, I”, Proc. Roy. Soc. Lond. A 92:638 (1916), 204-230.

[Spohn 1991] H. Spohn, Large scale dynamics of interacting particles, Springer, 1991.

[Stevens 2020] H. Stevens, "Why outbreaks like coronavirus spread exponentially, and how to 'flatten the curve", , The Washington Post (March 14, 2020).

[Wagner 1992] W. Wagner, "A convergence proof for Bird's direct simulation Monte Carlo method for the Boltzmann equation”, J. Statist. Phys. 66:3-4 (1992), 1011-1044.

Received 16 May 2020. Accepted 26 Jun 2020.

MARIO PULVIRENTI: pulvirenti@mat.uniroma1.it

Dipartimento di Matematica, Università di Roma La Sapienza, Rome, Italy

SERGIO SimOnELla: sergio.simonella@ens-lyon.fr

Unité de Mathématiques Pures et Appliquées, CNRS UMR 5669, École Normale Supérieure de Lyon, Lyon, France 